\title{
Solar Hot Air for Industrial Applications Using Linear Fresnel Concentrating Collectors and Open Brayton Cycle Layout
}

\author{
Antonio Famiglietti ${ }^{*}$, Antonio Lecuona-Neumann ${ }^{1}$, Mohammad Rahjoo $^{1}$, José Nogueira-Goriba ${ }^{2}$ \\ ${ }^{1}$ Universidad Carlos III de Madrid, Departamento de Ingenieria Termica y de Fluidos, Avda. de la Universidad 30, 28911 Leganes, \\ Madrid, Spain \\ ${ }^{2}$ Universidad de Castilla La Mancha, Campus de Excelencia Internacional en Energia y Medioambiente, Escuela de Ingenieria \\ Industrial y Aeroespacial, Av. Carlos III, s/n, Toledo 45071, Spain
}

\begin{abstract}
Many industrial processes use hot air as a medium. This is especially relevant for drying. Nowadays, most of them consume fossil energy. This study analyses an innovative facility for direct solar heating of air up to $400{ }^{\circ} \mathrm{C}$. For this aim, a medium-scale solar field of linear Fresnel collectors is proposed to heat ambient air inside it. No other dedicated heat transfer fluid is necessary. A simpler layout avoids costs, hurdles, and risks of heat transfer liquids and the related machinery. A Brayton cycle configuration avoids auxiliary energy consumption for pumping air through the collectors by coupling the solar field with a turbocharger. A mathematical model simulates a system of industrial size along a typical meteorological year. It confirms the feasibility of the proposal and the high potential of helping the energy transition to sustainability.
\end{abstract}

\section{Introduction}

The use of hot air is common to several industrial sectors, spanning from pulp and paper, manufacturing, minerals processing, to biomass and water residue treatments. Drying is one of the most widespread practices requiring air at different temperatures, according to the specific process and product treated [1], but pointing to medium temperatures. Drying is a much energy-consuming process that amounts up to $30 \%$ of heat consumption in Europe's industry sector.

Solar thermal technologies can play a vital role in replacing fossil fuel sources [2]. Flat plate collectors, evacuated tube collectors, and air collectors can generate hot air for industrial applications in the lowtemperature range, below $100{ }^{\circ} \mathrm{C}$. In the medium temperature range, up to $400{ }^{\circ} \mathrm{C}$, linear concentrating collectors are good candidates as a solar heat source. However, their application to air-based processes is not common [3], probably for the enormous convenience of directly heating by burning fuel or using electricity.

Famiglietti et al. [4] investigated the direct air heating inside a concentrating solar collector, eliminating the need for a dedicated heat transfer fluid (water, thermal oil, etc.) as well as an intermediate heat exchanger needed to deliver heat to process air. They theoretically analyzed an innovative direct solar air heater, which involves a Brayton cycle. The concept is of null work efficiency in mind, to avoid auxiliary pumping energy consumption. The present work extends the concept to a medium-scale solar field of Linear Fresnel Collectors, LFC, to investigate its technical feasibility under the solar variability.

\section{Turbo-assisted solar air heater}

Fig.1 shows the proposed layout of the Turbo-assisted Solar Air Heater T-SAH. $n_{p}$ U-loops in parallel form the solar field. Each U-loop consist of a series of $n_{s}$ LFC modules. The 4 U-loops here considered, are connected to a turbocharger, a unique device that joins a radial turbine $e$ and centrifugal compressor $c$ sharing a shaft. Ambient air is compressed up to 2 to 3 bar prior to entering the U-loop, where the absorbed concentrated irradiance heats it. The increased density allows reducing the mean flow velocity inside the receiver

\footnotetext{
* Corresponding author: antonio famiglietti@ live.it
} 
tubes, limiting the U-loop pressure drop. Heated air expands through the turbine $e$ before being delivered to the user. Under design operation, the turbine can provide the power required by the compressor to pump the flow so that the system is autonomous and the turbocharger runs in freewheeling mode. This concept corresponds to a Brayton cycle operation with null mechanical efficiency, delivering hot air in the range 300 to $400{ }^{\circ} \mathrm{C}$ at the turbine outlet. Auxiliary compressor $a c$ is active only during starting transient as well as for safety control purposes.

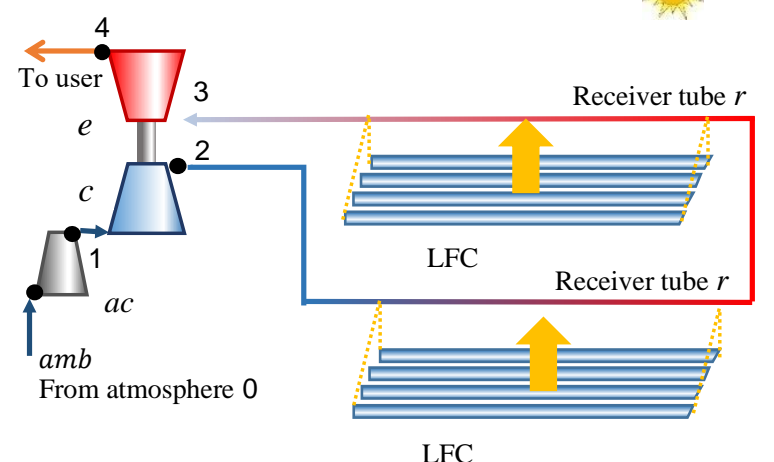

Fig. 1. Turbo-assisted solar air heater layout, with $\boldsymbol{n}_{\boldsymbol{s}}=2$ and $\boldsymbol{n}_{\boldsymbol{p}}=1$

\subsection{Solar field}

The solar field incorporates a commercial LFC [5], having an aperture $W_{a}=5.00 \mathrm{~m}$, length $L_{m}=5.28 \mathrm{~m}$ and receiver tube height over the mirrors plane $H_{m}=$ $2.72 \mathrm{~m}$. 24 LFC modules are connected forming $n_{p}=$ 4 U-loops, with $n_{s}=6 \mathrm{LFCs}$ in series. The total solar field reflecting area is $A_{t o t}=633.6 \mathrm{~m}^{2}$. The collectors' axes are oriented North-South. The receiver tube is evacuated and covered by a selective surface, having an internal diameter $D=0.066 \mathrm{~m}$. According to current practice in solar thermal engineering, [6], the optical efficiency of LFCs is modelled using transversal and longitudinal incident angle modifiers $I A M_{T}$, and $I A M_{L}$, provided by the manufacturer, as well as peak optical efficiency of $\eta_{o p 0}=0.632$. The factor $f_{\text {end }}$ accounts for the optical end losses due to concentrated irradiance impacting out of receiver length [7] so that the power gathered by the receiver tube results $\dot{Q}_{r}=\dot{Q}_{s} f_{\text {end }}$

$$
\begin{aligned}
& \dot{Q}_{b n}=G_{b n} A_{t o t} \\
& \dot{Q}_{s}=G_{b n} \eta_{o p 0} I A M_{T} I A M_{L} A_{\text {tot }} \\
& \dot{Q}_{r}=G_{b n} \eta_{o p 0} I A M_{T} I A M_{L} A_{\text {tot }} f_{\text {end }}
\end{aligned}
$$

More details on the receiver tube thermal model are given by [4].

\subsection{Turbocharger}

A commercial automotive turbocharger is selected from the available models to match the solar field characteristics. The compressor numerical model is obtained using continuous functional relations between the mass flow rate $\dot{m}_{a}$, the rotating speed $n_{T}$, the inlet temperature, the pressure ratio $\pi_{c}$, and the isoentropic efficiency $\eta_{c}$. Both, the flow model $\pi_{c}=p_{2} / p_{1}=$ $\pi_{c}\left\langle\dot{m}_{a}, T_{1}, n_{T}\right\rangle$, and the efficiency model $\eta_{c}=$ $\eta_{c}\left\langle\dot{m}_{a}, T_{1}, n_{T}\right\rangle$ are inter/extrapolated from performance maps provided by the manufacturer, [8]. The turbine flow functional model $\pi_{e}=\frac{p_{3}}{p_{4}}=\pi_{e}\left\langle\dot{m}_{a}, T_{3}\right\rangle$ is also inter/extrapolated from the flow map. The isoentropic efficiency $\eta_{e}=\eta_{e}\left\langle\dot{m}_{a}, T_{3}, n_{T}\right\rangle$ is modelled theoretically following this work [9].

\subsection{Numerical simulation}

According to the input conditions, such as ambient temperature $T_{a m b}$, direct normal solar irradiance $G_{b n}$, sun azimuth and elevation, the quasi-steady-state numerical model allows determining the main variables that describe the operational status of the system: air pressure and temperature at any point of the cycle in Fig. 1, the mass flow rate $\dot{m}_{a}$, turbocharger speed $n_{T}$, thermal power gathered by the receiver tubes $\dot{Q}_{r}$, the thermal losses to ambient $\dot{Q}_{L}=\dot{Q}_{r}-\dot{Q}_{u}$, the optical end losses $\dot{Q}_{\text {fend }}=\dot{Q}_{s}-\dot{Q}_{r}$, the power delivered to the user $\dot{Q}_{a}$, among other parameters.

$$
\begin{aligned}
& \dot{Q}_{u}=\left(T_{3} c_{p, 3}-T_{2} c_{p, 2}\right) \dot{m}_{a} \\
& \dot{Q}_{a}=\left(T_{4} c_{p, 4}-T_{a m b} c_{p, a m b}\right) \dot{m}_{a}
\end{aligned}
$$

The thermal inertia of the solar receiver, piping, and turbocharger is not taken into consideration by the model. The system is simulated as a sequence of quasisteady-states corresponding time steps of one hour. Using a typical meteorological year TMY [10] of Madrid (Spain), a simulation is run all over the year to evaluate the T-SAH performances.

\section{Results}

The T-SAH concept simulation along the TMY for the selected location (Madrid) reveals its operating range. The system can operate in a wide range of solar irradiances available during the year, confirming that the matching between the solar field and the turbocharger is good. For moderate and high solar irradiances, the turbocharger can run freewheeling since the turbine power balances the compressor consumption. Along the days, the output temperature $T_{4}$ varies between $300{ }^{\circ} \mathrm{C}$ and $400{ }^{\circ} \mathrm{C}$ according to the aim of the technology. The hot air mass flow rate delivered to the thermal process ranges between $0.2 \mathrm{~kg} / \mathrm{s}$ and $0.7 \mathrm{~kg} / \mathrm{s}$. Figure 2 reports the output of T-SAH in terms of air temperature and mass flow rate during the operating hours of TMY. The peak temperature reached by the receiver tube does not exceed the thermal limit recommended by the manufacturers, $T_{w, \max }=600^{\circ} \mathrm{C}$. 


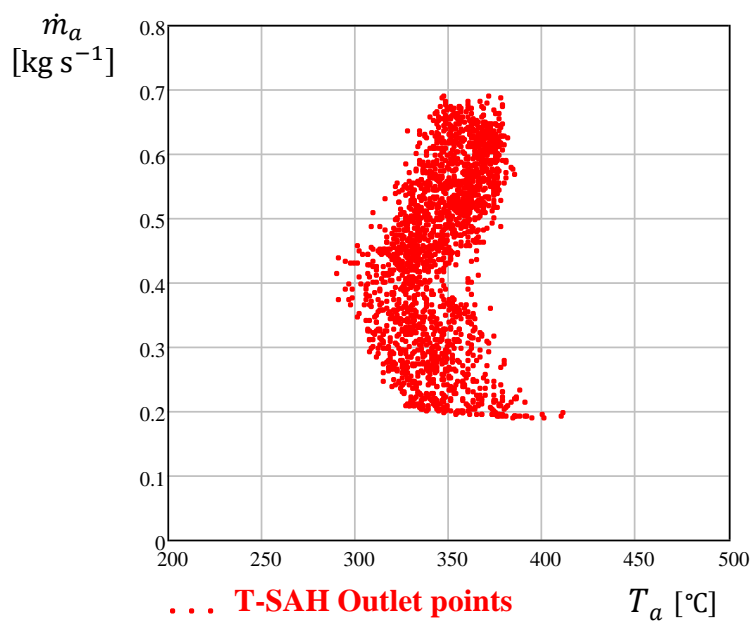

Fig. 2. Air temperature and mass flow rate delivered to the thermal process during the operating hours of TMY.

Figure 3 shows the various energy contributions on a monthly basis during the operating hours, according to Eqs. (1), (2) and (3).

The thermal energy delivered to the airflow sum the hourly energy contributions from the first to the last $i$ hour of the month when the T-SAH is ON, $Q_{a}=$ $\sum \dot{Q}_{a_{i}}$ hr. Figure 3 reports the thermal losses $Q_{L}=$ $\sum \dot{Q}_{L_{i}} \mathrm{hr}$ as well as optical end losses $Q_{\text {fend }}=$ $\sum \dot{Q}_{\text {fend }_{i}}$ hr. Normal direct (beam) solar energy available is computed considering the operating hours of the T-SAH, $Q_{b n}=\sum \dot{Q}_{b n_{i}}$ hr. Both concentrated energy on the focal line, $Q_{s}=\sum \dot{Q}_{s_{i}} \mathrm{hr}$, and on the receiver $Q_{r}=\sum \dot{Q}_{r_{i}} \mathrm{hr}$ are indicated, as well as optical losses $Q_{o p}=\sum\left(\dot{Q}_{b n_{i}}-\dot{Q}_{s_{i}}\right) \mathrm{hr}$.

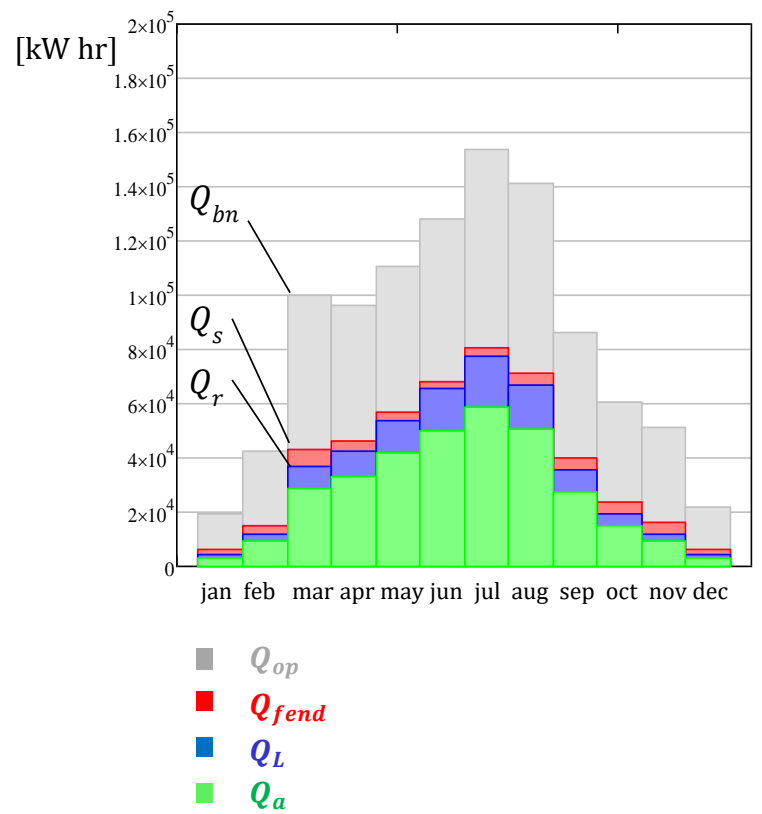

Fig. 3. Monthly energy contributions across de TMY

Along the year, the T-SAH delivers $330 \mathrm{MW} \mathrm{hr}$ of thermal energy in the range of temperature 300 to 400
${ }^{\circ} \mathrm{C}$, working for $\sim 2,000 \mathrm{hr}$ per year, with a specific

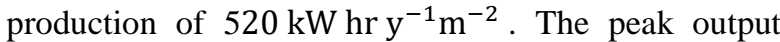
power is $265 \mathrm{~kW}_{\mathrm{th}}$, corresponding to $415 \mathrm{~W}_{\mathrm{th}} \mathrm{m}^{-2}$, with an annual average efficiency $\bar{\eta}_{T S A H}=\frac{\dot{Q}_{a}}{\dot{Q}_{b n}}=0.33$.

For very low solar irradiances, the T-SAH can not operate. The turbocharger mechanical balance is not accomplished due to the minimum rotating speed limit of the compressor and the drop of compressor and turbine efficiencies occurring when the turbocharger operates out of its design point. These conditions are expected to take place during the early morning and late afternoon, especially in the winter season, and less extensive in summer. However, the loss of monthly and annual energy due to low irradiance cut-off is limited, as shown in Figure 4. The monthly energy gathered by the receiver tube $Q_{r}$ during the working hours is shown, together with the energy not exploited during the hours the T-SAH is OFF, due to low irradiance cut-off.

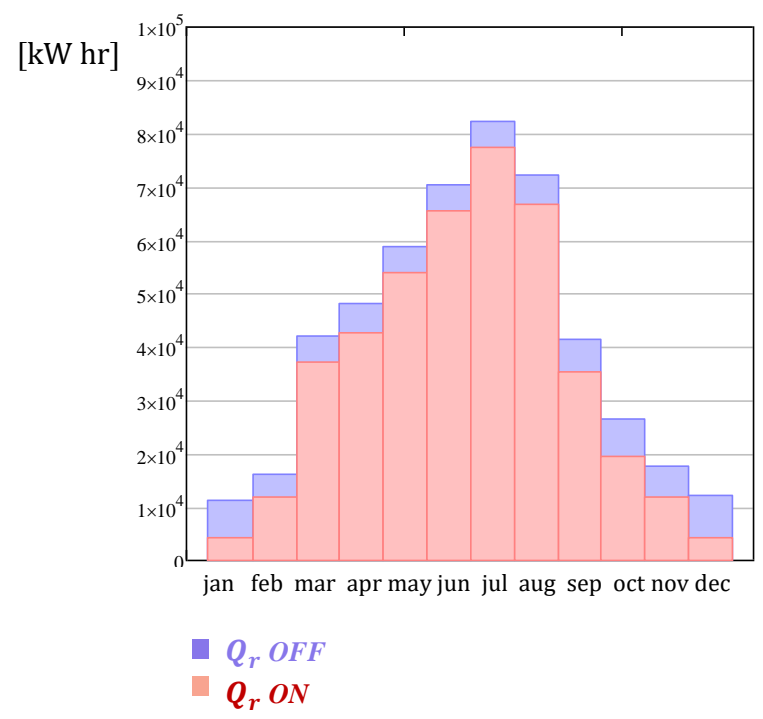

Fig. 4. Energy gathered by the receiver tube with the T-SAH during ON hours and energy not exploited during OFF hours along each month.

Although the amount of solar energy lost during low irradiance cut-off hours is relatively low, a modest additional annual energy yield could be achieved by extending the operative range of the system. Alternatives to the presented T-SAH operation mode can be considered at low irradiance although they are not investigated in this work. One possibility is to use the auxiliary compressor $a c$ is series with the compressor $c$ to provide the additional mechanical power required by the turbocharger to run steady. Another possibility is excluding the turbocharger from the circuit, blowing air through the solar field using the auxiliary compressor $a c$ only. In both cases, the low mass flow rate required at low solar irradiance and the consequent moderate stagnation pressure drops across the air circuit suggest an acceptably small auxiliary energy consumption. 


\section{Conclusions}

In this study an innovative layout for direct solar air heating is proposed. The use of concentrating linear Fresnel collector allows to reach higher temperature in relation to other non-concentrating solar technologies. A solar Brayton cycle is implemented coupling the solar field with a turbocharger, aiming to avoid auxiliary consumption for pumping air through the solar field, ensuring a delivery temperature in the medium temperature range. The numerical simulation carried out demonstrates the technical viability of the concept and encourage further research.

\section{References}

[1] G. Pirasteh, R. Saidur, S. M. A. Rahman, and N. A. Rahim, "A review on the development of solar drying applications," Renew. Sustain. Energy Rev., vol. 31, pp. 133-148, 2014.

[2] S. H. Farjana, N. Huda, M. A. P. Mahmud, and R. Saidur, "Solar process heat in industrial systems - A global review," Renew. Sustain. Energy Rev., vol. 82, no. August 2017, pp. 2270-2286, 2018.

[3] A. K. Sharma, C. Sharma, S. C. Mullick, and T. C. Kandpal, "Solar industrial process heating: A review," Renew. Sustain. Energy Rev., vol. 78, no. December 2016, pp. 124137, 2017.

[4] A. Famiglietti, A. Lecuona-Neumann, J. Nogueira, and M. Rahjoo, "Direct solar production of medium temperature hot air for industrial applications in linear concentrating solar collectors using an open Brayton cycle. Viability analysis," vol. 169, no. September 2019, 2020.

[5] Solatom, "SOLAR STEAM FOR INDUSTRIAL PROCESSES." [Online]. Available: http://www.solatom.com/.

[6] S. Karathanasis, Linear Fresnel Reflector Systems for Solar Radiation Concentration. 2019.

[7] A. Heimsath, G. Bern, D. Van Rooyen, and P. Nitz, "Quantifying optical loss factors of small linear concentrating collectors for process heat application," Energy Procedia, vol. 48, no. September 2013, pp. 77-86, 2014.

[8] L. Guzzella and C. H. Onder, Introduction to modeling and control of internal combustion engine systems. 2010.

[9] S. A. Frei, L. Guzzella, C. H. Onder, and C. Nizzola, "Improved dynamic performance of turbocharged SI engine power trains using clutch actuation," Control Eng. Pract., vol. 14, no. 4, pp. 363-373, 2006.

[10] A. Habte et al., "Best Practices Handbook for the Collection and Use of Solar Resource Data for Solar Energy Applications: Second
Edition," NREL, no. December 2017, pp. 2.12.22, 2017. 